

\section{Sumário}

I. Crônicas do Direito Internacional ...............................................1

REPARAÇÃo DE VÍTIMAS À LUZ DE UM TRATAdo SOBRE EMPRESAS E DIREITOS HUMANOS ............ 3 Ana Cláudia Ruy Cardia

Consumer Social Responsibility as a Requirement for Corporate Social ResponsibiliTY

Nitish Monebhurrun

Crisis in Venezuela: The Brazilian response to the massive flow of Venezuelans in

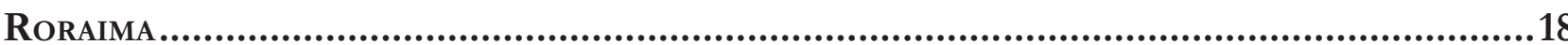

Jacqueline Salmen Raffoul

II. Dossiê EsPecial: Business and Human Rights.........................................23

Some remarks on the third sessions of the Business and Human Rights Treaty Pro-

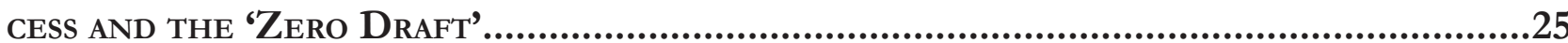

Humberto Cantú Rivera

The United Nations guiding principles on business and human Rights, the State

DUTY TO PROTECT HUMAN RIGHTS AND THE STATE-BUSINESS NEXUS.

Mihaela Maria Barnes

HARdening SOFT LAW: ARE THE EMERging Corporate SOCIAL DisClOSURE LAWS CAPABLE OF GENERATING SUBSTANTIVE COMPLIANCE WITH HUMAN RIGHTS?

Justine Nolan

Del Documento de Elementos al Draft 0: apuntes jurídicos respecto del posible CONTENIDO DEL PROYECTO DE INSTRUMENTO VINCULANTE SOBRE EMPRESAS TRANSNACIONALES

Y OTRAS EMPRESAS CON RESPECTO A LOS DERECHOS HUMANOS

Adoración Guamán 
ACCESS TO REMEDIES AND THE EMERGING ETHICAL DILEMMAS: CHANGING CONTOURS WITHIN THE BUSINESS-HUMAN RIGHTS DEBATE

Justin Jos

LA RESPONSABILIDAD PENAL DE LAS EMPRESAS POR GRAVES VIOLACIONES DE DERECHOS HUMANOS: PRÁCTICA ACTUAL Y DESAFÍOS FUTUROS 130

Daniel Iglesias Márquez

THE ENVIRONMENTAL LAW DIMENSIONS OF AN INTERNATIONAL BINDING TREATY ON BUSINESS AND HUMAN RIGHTS

Juan Gabriel Auz Vaca

Los Objetivos de Desarrollo Sostenible en Europa y su Intersección con el Marco de los Negocios y los Derechos Humanos

Paolo Davide Farah

HumAN RIGHTS AND MARKET ACCESS 203

Danielle Mendes Thame Denny

BusinesS AND HUMAN RIGHTS IN BRAZIL: EXPLORING HUMAN RIGHTS DUE DILIGENCE AND OPERATIONAL-LEVEL GRIEVANCE MECHANiSMS IN THE CASE OF KinRoss PARACATU Gold Mine...222 Mariana Aparecida Vilmondes Türke

Human Rights and eXtractive industries in Latin America: What Responsibility of CORPORATIONS AND THEIR STATES OF ORIGIN FOR HUMAN RIGHTS VIOLATIONS IN THE INTER-American Human Rights System?.

Alberto do Amaral Junior e Viviana Palacio Revello

MultinaCiOnAIS FAST FASHION E DIREITOS HUMANOS: EM BUSCA DE NOVOS PADRÕES DE RESPONSABILIZAÇÃO 255

Laura Germano Matos e João Luis Nogueira Matias

III. Artigos sobre outros temas

EFFICIENCY AND EFFICACY OF PUBLIC FOOD PROCUREMENT FROM FAMILY FARMERS FOR SCHOOL FEEDING IN BRAZIL. 271

Rozane Márcia Triches 
A relaÇão ENTRE O GRAU de INTEGRaÇÃo ECONÔMiCA E O SISTEMA DE SOLUÇÃo DE CONTROvÉRSIAS: UM ESTUdo COMPARATIVO ENTRE A UNIÃo EURopeia E O MERCOSUl.....................286

Luciane Klein Vieira e Elisa Arruda

THE RIGHTS TO MEMORY AND TRUTH IN THE INTER-AMERICAN PARADIGMS OF TRANSITIONAL JUSTICE: THE CASES OF BRAZIL AND CHILE 308

Bruno Galindo

Juliana Passos de Castro

A margem nacional de apreciação na Corte Interamericana de Direitos Humanos.325 Gilberto Schäfer, José Eduardo Aidikaitis Previdellie e Jesus Tupã Silveira Gomes

Novos Direitos FUNDAMENTAIS NO ÂMBITo DA UNASUL: ANÁLISE DAS AGENDAS DE Brasil E VENEZUELA À LUZ DO DIREITO À PAZ.

Pedro Pulzatto Peruzzo e Arthur Ciciliati Spada

A atuação do Grupo Mercado Comum frente À Criminalidade organizada transnacio-

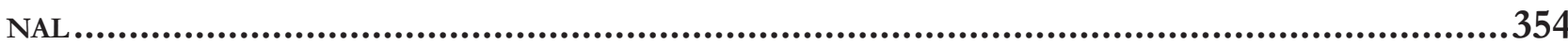

Sabrina Cunha Kesikowski, Luis Alexandre Carta Winter e Eduardo Biacchi Gomes

COUNTER-TERRORISM LEGISLATION AND TERRORIST ATTACKS: DOES HUMAN RIGHTS HAVE SPACE?

Heloisa Tenello Bretas e Daniel Damásio Borges

Territórios da ViolênCia de GÊNERo: NORMATIVA INTERNACIONAL E os Casos "CAMpo AlGodoeiro” (MÉXICO) - “Morro do Garrote” (BrasiL)

Eugênia Nogueira do Rêgo Monteiro Villa e Bruno Amaral Machado

O USO DE MECANISMOS INFORMAIS DE GOVERNANÇA GLOBAL E SUA APLICABILIDADE NAS LICITAÇÕES PÚBLICAS BRASILEIRAS.

Fabiano de Figueiredo Araujo e Paulo Afonso Cavichioli Carmona

Autonomia INSTITUCIONAL DA IGREJA CATÓliCA E A INGERÊNCIA INDEVIDA DO ESTADO BRASILEIRo POR EVENTUAIS Ilícitos CANÔNicos: ANÁlise do CASO de Formosa-GO, À luz do TraTADo Brasil-Santa SÉ de 2010 
A MORE TARGETED APPROACH TO FOREIGN DIRECT INVESTMENT: THE ESTABLISHMENT OF SCREENING SYSTEMS ON NATIONAL SECURITY GROUNDS

Carlos Esplugues Mota

IV. RESENHAS 467

Direito Internacional em Perspectiva Transcivilizacional de Yasuaki Onuma........469 Arthur Roberto Capella Giannattasio

Resenha do livro Space, Global Life: The Everyday Operation of International LaW and Development, de Luis Eslava .473

Matheus Gobbato Leichtweis

QUEM TEM MEDO DO PÓS-COLONIAL NO DIREITO INTERNACIONAL? UMA RESENHA DE "DECOLONISING INTERNATIONAL LAW: DEVELOPMENT, ECONOMIC GROWTH AND THE POLITICS OF UNIVERSAlity" de Sundhya Pahuja

Gabriel Antonio Silveira Mantelli

Direitos humanos COMO UM NOVO PROJETO PARA O Direito INTERNACIONAL? Notas sobre The Last Utopia, de Samuel Moyn .490 João Roriz 


\title{
La responsabilidad penal de las empresas por graves violaciones de derechos humanos: práctica actual y desafíos futuros*
}

\author{
Corporate criminal liability for gross human \\ rights violations: current practice and future \\ challenges
}

Daniel Iglesias Márquez**

\section{Resumen}

La vía penal, tanto en su vertiente internacional como nacional, constituye otro posible mecanismo judicial para responsabilizar a las empresas o, en su defecto, a los directivos o ejecutivos de las mismas por la vulneración de derechos humanos. La responsabilidad penal de las empresas es aún un tema controversial y adopta diversos enfoques en cada Estado. Por tanto, el Derecho penal ha recibido menos atención que otras vías y, en consecuencia, su potencial para responsabilizar a las empresas por sus impactos sociales y ambientales no ha sido suficientemente explorado. A partir del análisis descriptivo y comparativo de la práctica actual y de la bibliografía relacionada con el tema se examina el potencial y los obstáculos legales, políticos y prácticos a superarse para hacer efectiva la responsabilidad penal por los graves abusos de derechos humanos cometidos en el marco de las actividades globales de las empresas transnacionales.

Palabras clave: Derecho penal. Empresas y derechos humanos. Violaciones de derechos humanos. Tercer pilar de los Principios Rectores sobre empresas y derechos humanos.

* Recebido em 13/06/2018

Aprovado em 17/08/2018

** Doctor en Derecho por la Universidad Rovira i Virgili de Tarragona (España). Investigador asociado al Centro de Estudios de Derecho Ambiental de Tarragona (CEDAT-URV) y miembro de la Red de Empresas, Conflicto y Derechos Humanos (BCHR Network). Email: daniel.iglesias@urv.cat

\section{Abstract}

Criminal law, both internationally and nationally, is another judicial mechanism to hold corporations -or corporate agents- accountable for human rights violations. Corporate criminal liability is still contested and each State has adopted a different approach. Thus, criminal law has received less attention than other avenues, and hence its high potential to hold corporations accountable for their social and environmental impacts has not been sufficiently tested. Thus, taking into account the current practice and the bibliography related to the theme, this article examines through a comparative and descriptive analysis the potential and the legal, political and practical obstacles for holding transnational corporations criminally liable for gross human rights abuses committed in the framework of their global activities.

Keywords: Criminal Law. Business and Human Rights. Human rights violations. Third pillar of the Guiding Principles on Business and Human Rights. 


\section{INTRODUCCIÓN}

Durante los casi cincuenta años de debate sobre el tema de empresas y derechos humanos, una de las cuestiones en la agenda internacional a la que se ha intentado dar solución es a la búsqueda de mecanismos efectivos para responsabilizar a las empresas por las graves violaciones de derechos humanos cometidas en el desarrollo de sus actividades. A día de hoy, la comunidad internacional no ha logrado alcanzar un consenso en la creación de un mecanismo que permita alcanzar de manera efectiva este objetivo. Existen propuestas desde la academia y de la sociedad civil para crear un tribunal internacional sobre empresas y derechos humanos que permita acceder a las personas afectadas a una instancia judicial internacional independiente. No obstante, aún quedan cuestiones pendientes sobre los recursos humanos y financieros que requiere para su funcionamiento. ${ }^{1}$

En este orden de ideas, le corresponde a los Estados la carga de garantizar el acceso a los recursos judiciales existentes para las víctimas de violaciones de derechos humanos cometidas por las empresas. A día de hoy, las víctimas pueden recurrir a diversos mecanismos para hacer efectiva la responsabilidad de las empresas y recibir una reparación justa por los daños sufridos, aunque cada mecanismo tiene resultados y alcances diferentes. Por tanto, el panorama actual deja entrever que, por lo general, las víctimas se adentran en un laberinto de mecanismos judiciales -y no judiciales- con diversos obstáculos a superar.

El Derecho penal constituye uno de los mecanismos judiciales a los que las víctimas pueden recurrir. Algunos progresos y casos recientes demuestran que la vía penal, tanto en su vertiente internacional como nacional, presenta importantes oportunidades para responsabilizar a las empresas por graves violaciones de derechos humanos. Así, el Derecho penal podría llevar el debate sobre empresas y derechos humanos a nuevos horizontes, no obstante, a diferencia de otros mecanismos, esta vía ha recibido menos atención, manteniendo de esta manera los obstáculos doctrinales y legales que hasta el momento parecían inamovibles.

1 HERNÁNDEZ ZUBIZARRETA, Juan. El Tribunal Internacional para las empresas transnacionales y los derechos humanos. América Latina en movimiento, p. 14-15, 2016; Ramasastry, Anita; CASSELL, Douglass. White Paper: Options for a Treaty on Business and Human Rights. Notre Dame Journal of International \& Comparative Law, v. 6, n. 1 , p. $1-50,2015$.
El presente artículo, a través de un análisis comparativo y descriptivo de la práctica actual, examina las oportunidades y el potencial de la vía penal, tanto a nivel internacional como nacional, como mecanismo judicial para hacer efectiva la responsabilidad de las empresas por graves violaciones de derechos humanos. El objetivo principal del presente artículo es aportar elementos que permitan determinar la viabilidad del Derecho penal y la justicia penal como un mecanismo judicial a ser contemplado y reforzado en las disposiciones del futuro tratado sobre empresas y derechos humanos o, en su defecto, en la implementación del tercer pilar de los Principios Rectores de las Naciones Unidas sobre empresas y derechos humanos a nivel nacional. Para ello, se aportan, en primer lugar, algunas reflexiones preliminares sobre el alcance de la vía penal en el debate sobre empresas y derechos humanos. Posteriormente se analizan las oportunidades actuales y los avances para recurrir al Derecho penal internacional para hacer efectiva la responsabilidad de las empresas por graves violaciones de derechos humanos. Seguidamente, se profundiza en la experiencia y los obstáculos prácticos y políticos del enjuiciamiento penal a nivel nacional para luchar contra la impunidad de los delitos corporativos relacionados con abusos de derechos humanos. Finalmente, se concluye que la justicia penal es clave en el debate sobre empresas y derechos humanos y, sobre todo, que la actual coyuntura se presta para que los Estados avancen en el desarrollo de esta vía en aras de adoptar las medidas legislativas y políticas para paliar los obstáculos en el enjuiciamiento penal de las empresas, tanto a nivel internacional como nacional.

\section{LA VÍA PENAL COMO MECANISMO JUDICIAL PARA RESPONSABILIZAR A LAS EMPRESAS POR LA VULNERACIÓN DE DERECHOS HUMANOS}

En la práctica y en la doctrina legal, ${ }^{2}$ la vía civil ha

2 Existe una abundante doctrina legal relacionada con la práctica del litigio civil en los Estados Unidos contra las empresas por la vulneración de derechos humanos. No obstante, queda fuera del análisis del presente artículo. Algunos autores que abordan el litigio civil transnacional en los Estados Unidos contra empresas por violaciones de derechos humanos en tercero Estados, véase, REQUEJO ISIDRO, Marta. Responsabilidad civil y derechos humanos en EEUU: ¿el fin del ATS? InDret. Revista para el análisis del Derecho, n. 3, p. 1-38, 2011; STEPHENS, Beth. Corporate Accountability: International Human Rights Litigation against Corporations in US Courts. En: KAMMINGA, Menno T.; ZIA-ZARIFI, Saman (Ed.). Liability of Multinational Corporations under International Law. La Haya-Londres-Boston: Kluwer Law International, 2000. p. 209-230. 
sido el mecanismo judicial más explorado y recurrido hasta el momento para responsabilizar a las empresas por violaciones de derechos humanos. Sin embargo, las víctimas y las organizaciones de la sociedad civil (OSC) instan cada vez más a los Estados al ejercicio de la jurisdicción penal para investigar y enjuiciar a las empresas por las graves violaciones de las normas internacionales de derechos humanos, de Derecho humanitario y de Derecho penal internacional. Por ejemplo, a raíz de una investigación realizada por Amnistía Internacional sobre la campaña de la empresa Shell para silenciar las protestas en Ogoniland en la década de los noventa, la organización solicitó a los gobiernos de Nigeria, de los Países Bajos y del Reino Unido una investigación penal para determinar la complicidad de la empresa en delitos relacionados con violaciones de derechos humanos cometidas por las fuerzas de seguridad nigerianas. ${ }^{3}$

Tradicionalmente, la doctrina penal clásica se ha mostrado reacia al reconocimiento de la responsabilidad penal de las personas jurídicas debido a que estos entes, considerados ficticios, carecen por su propia naturaleza de las actitudes mentales (mens rea) - ya sean intencionales, imprudentes o negligentes- para la comisión de delitos, lo que se constituye como un elemento de la responsabilidad penal. Asimismo, la negativa a reconocer la responsabilidad penal de las personas jurídicas se debe también, entre otras cuestiones, a las diferencias en los modelos de atribución de responsabilidad, a la determinación del grado de culpabilidad colectiva o al tipo de sanciones aplicables a las personas jurídicas.

Sin embargo, la limitación histórica y clásica de los sistemas jurídicos penales en relación a las personas jurídicas presenta una evolución relevante encaminada a la lucha contra la delincuencia empresarial. Esto ha servido para superar los dogmas individualistas y personalistas característicos del esquema tradicional del Derecho penal. Y, a su vez, para la construcción de un sistema de responsabilidad penal de las personas jurídicas, dejando atrás el dogma de "societas delinquere non potest", pero sin llegar a ser este tipo de responsabilidad penal universalmente aceptada por todos los Estados.

En este punto, cabe advertir que la responsabilidad penal de las empresas no significa que los responsables individuales en el seno de las personas jurídicas no pue-

3 Amnistía Internacional. A Criminal Enterprise? Shell's Involvement in Human Rights Violations in Nigeria in the 1990s. Londres: Amnesty International, 2017. p. 89. dan ser enjuiciados y sancionados penalmente cuando se intente desplazar la responsabilidad a las entidades ficticias, salvo que diera lugar a castigos desproporcionados por un mismo delito. La responsabilidad penal de las empresas puede y debe existir junto a la responsabilidad de los agentes corporativos. No obstante, el presente artículo pone un mayor énfasis en la responsabilidad penal de las empresas, solo haciendo referencia a la responsabilidad de sus directivos y ejecutivos en la medida que se considere oportuno, ya que, como se analiza en los siguientes apartados, forma parte de la práctica actual para hacer efectiva la responsabilidad de los sujetos implicados en violaciones de derechos humanos cometidas en el desarrollo de las actividades empresariales.

Así, el Derecho penal juega un papel cada vez más importante para garantizar que las empresas respondan por sus actos ante la sociedad y para prevenir su impunidad, ya que se constituye como una vía disponible para responsabilizar a las empresas cuando cometan o participan en graves abusos de derechos humanos.

En 2011, el Consejo de Derechos Humanos de las Naciones Unidas adoptó por unanimidad los Principios Rectores sobre Empresas y Derechos Humanos (Principios Rectores), elaborados entre 2005 y 2011 por el Representante Especial del Secretario General para la cuestión de los derechos humanos y las empresas, John Ruggie. Los Principios Rectores rigen actualmente el vínculo entre empresas y derechos humanos. Este instrumento cuenta con un enfoque predominantemente de carácter soft law y desarrolla un sistema interrelacionado de medidas de prevención y reparación de violaciones de derechos humanos cometidas por empresas.

Los Principios Rectores advierten que a día de hoy existe un riesgo para las empresas de incurrir en una responsabilidad penal por violaciones de derechos humanos. En primer lugar, el Principio 7 aborda la obligación de los Estados de asegurar que las empresas que operan en zonas afectadas por conflictos no se vean implicadas en violaciones graves de derechos humanos, adoptando las medidas adecuadas que pueden explorar las responsabilidades civiles, administrativas o penales de las empresas domiciliadas u operativas en su territorio y/o jurisdicción que cometan o participen en este tipo de abusos.

Más adelante, el Comentario al Principio 17 señala que pueden generarse supuestos de complicidad cuando una empresa contribuye o parece contribuir a las conse- 
cuencias negativas sobre los derechos humanos causadas por otras partes. La complicidad implica una responsabilidad penal en varias jurisdicciones nacionales.

Finalmente, el Comentario al Principio 23 destaca que varios Estados que reconocen la responsabilidad penal de las personas jurídicas han incorporado en sus ordenamientos internos las disposiciones del Estatuto de Roma de la Corte Penal Internacional (Estatuto de Roma). En este sentido, algunos Estados, como Italia o Dinamarca, proponen ya en sus Planes de Acción Nacional sobre empresas y derechos humanos medidas relacionadas con el Derecho penal que se alienan a lo señalado en los Principios Rectores.

Aún más específicos, algunos instrumentos internacionales e informes de expertos, como la Resolución del Parlamento Europeo sobre la responsabilidad de las empresas por violaciones graves de los derechos humanos en terceros países (2015/2315(INI)), la Recomendación CM/Rec (2016)3 del Comité de Ministros del Consejo de Europa sobre derechos humanos y empresas y el informe de los Corporate Crimes Principles, hacen un llamado a los Estados a recurrir al Derecho penal para hacer frente a los abusos corporativos y para abordar los obstáculos legales, procesales y prácticos que implica esta vía judicial.

La relevancia de la vía penal ha sido también parte del debate en los períodos de sesiones del Grupo de Trabajo Intergubernamental (GTI) encargado de elaborar el tratado vinculante sobre empresas y derechos humanos. Algunas delegaciones señalan que la máxima disuasión para que las empresas no cometan violaciones de derechos humanos se alcanzaría imponiendo la responsabilidad penal. ${ }^{4}$ Así, este futuro instrumento debería, por un lado, sentar las bases para que los Estados incorporen en la legislación penal nacional las formas de conducta que afectan el disfrute de los derechos humanos, incluidas las que ya están reconocidas por el Derecho internacional. ${ }^{5}$ Por otro lado, debería contribuir a

4 Consejo de Derechos Humanos, Informe del segundo período de sesiones del grupo de trabajo intergubernamental de composición abierta sobre las empresas transnacionales y otras empresas con respecto a los derechos humanos, A/HRC/34/47 (4 de enero de 2017), párr. 29.

5 Consejo de Derechos Humanos, Informe del primer período de sesiones del grupo de trabajo intergubernamental de composición abierta sobre las empresas transnacionales y otras empresas con respecto a los derechos humanos, con el mandato de elaborar un instrumento internacional jurídicamente vinculante, A/HRC/31/50 (5 de febrero de 2016), párr. 90. aclarar la responsabilidad penal de las personas jurídicas con el fin de alentar a que más Estados reconozcan este tipo de responsabilidad, sin excluir la de los directores y ejecutivos, para así poder combatir la impunidad y proteger los derechos de las víctimas de violaciones de derechos humanos. ${ }^{6}$

En este sentido, algunas OSC, de manera más contundente, abogan por que los Estados estén obligados a aprobar legislaciones que definan adecuadamente la responsabilidad penal para sancionar a las empresas que vulneren derechos humanos o contribuyan a ello. ${ }^{7} \mathrm{No}$ obstante, este tipo de obligación puede ser contraproducente debido a que algunos Estados pueden hacer reservas a dicha disposición, ya que, como se observa más adelante, no todas las jurisdicciones reconocen la responsabilidad penal de las personas jurídicas. Por tanto, se afectaría la facultad discrecional de los Estados de decidir el tipo de responsabilidad según los principios jurídicos de sus ordenamientos internos. La solución más práctica, teniendo en cuenta el panorama actual, sería que el futuro instrumento alentara a los Estados a que adoptaran las medidas normativas y otras iniciativas para establecer la responsabilidad penal o, en su defecto, la responsabilidad equivalente (civil o administrativa), de acuerdo a los principios jurídicos de cada Estado. ${ }^{8}$

La vía penal cumple principalmente una función preventiva, pues la posibilidad de que las empresas incurran en una responsabilidad penal tiende a incentivarlas a adoptar las medidas necesarias para prevenir abusos de derechos humanos, pues la posibilidad de que sean identificadas como culpables de un delito las estigmatiza. Un posible enjuiciamiento penal podría afectar directamente la imagen y la reputación de las empresas, condicionando así su acceso a los mercados mundiales y la movilidad de sus activos. Asimismo, las sanciones

6 Véase, "Elementos para el proyecto de instrumento internacional jurídicamente vinculante sobre empresas transnacionales y otras empresas con respecto a los derechos humanos." Disponible en: www.ohchr.org/Documents/HRBodies/HRCouncil/ WGTransCorp/Session3/LegallyBindingInstrumentTNCs_OBEs_ SP.pdf, p. 8-9.

7 Consejo de Derechos Humanos, Informe del primer período de sesiones del grupo de trabajo intergubernamental de composición abierta sobre las empresas transnacionales y otras empresas con respecto a los derechos humanos, con el mandato de elaborar un instrumento internacional jurídicamente vinculante, A/HRC/31/50 (5 de febrero de 2016), párr. 88.

8 ICJ. Proposals for Elements of a Legally Binding Instrument on Transnational Corporations and Other Business Enterprises. Ginebra: ICJ, 2016. p. 19-22. 
penales aplicables a la comisión de un delito pueden ser en ocasiones más onerosas. ${ }^{9}$ Así, aunque carezca de una función reparadora, la vía penal permite exponer ciertas prácticas empresariales que deben ser perseguidas y sancionadas por los Estados y, en consecuencia, influir en el comportamiento de las empresas.

Por consiguiente, esta vía constituye otro de los recursos judiciales existentes para hacer efectiva la responsabilidad de las empresas por graves violaciones de derechos humanos. No obstante, presenta importantes obstáculos sustantivos y procesales que reducen las posibilidades de que las víctimas y las OSC recurran a esta vía, tanto a nivel internacional como nacional. Dichos obstáculos se analizan en los siguientes apartados.

\section{EL ENJUICIAMIENTO DE LAS EMPRESAS POR LA VULNERACIÓN DE DERECHOS HUMANOS CONFORME al Derecho penal internacional}

Las más graves violaciones de derechos humanos pueden dar lugar a crímenes internacionales reconocidos en el Derecho penal internacional. En este sentido, algunas conductas delictivas graves como asesinatos, explotación sexual, esclavitud, tortura o desplazamientos forzados pueden llegar constituirse como crímenes de lesa humanidad. ${ }^{10}$ Por tanto, cabe plantearse en qué medida este tipo de conductas, cometidas en el marco de las actividades empresariales, activan la jurisdicción de los tribunales penales internacionales.

Cabe comenzar señalando que tras la Segunda Guerra Mundial la responsabilidad penal internacional se expandió a las personas físicas a través del Estatuto de Londres y de la jurisprudencia del Tribunal de Núremberg, con especial referencia a los casos contra los empresarios que participaron en la comisión de crímenes de genocidio y de guerra.

Es bien sabido que durante la Segunda Guerra Mundial las empresas alemanas cometieron graves atroci-

9 SMITH, Susan L. Changing Corporate Environmental Behaviour: Criminal Prosecutions as a Tool of Environmental Policy. En: ECKERSLEY, Robyn (Ed.). Markets, the State and the Environment towards Integration. Melbourne: McMillan Education Australia, 1995. p. 261-274.

10 PÉREZ-LEÓN ACEVEDO, Juan Pablo. The Close Relationship between Serious Human Rights Violations and Crimes against Humanity: International Criminalization of Serious Abuses. Anuario Mexicano de Derecho Internacional, v. 17, p. 145-186, 2017. dades. Los juicios posteriores al conflicto, iniciados en virtud de la Ley del Consejo de Control Aliado núm. 10, exploraron y debatieron por primera vez la delincuencia empresarial, creando un precedente importante en la responsabilidad penal internacional de las empresas. El Estatuto del Tribunal Militar Internacional de Núremberg le concedía competencias para declarar que un grupo u organización era una organización criminal, no obstante, solo las personas físicas pertenecientes a dicha organización podían ser enjuiciadas y sentenciadas (artículo 9 y 10).

Los casos más conocidos son en los que se acusó a varios empresarios y banqueros de complicidad en los crímenes cometidos durante la guerra (Flick, ${ }^{11}$ I.G. Farben ${ }^{12}$ y Krup $\left.p^{13}\right)$. Estos casos representan el primer precedente en el que se criminalizó a las empresas por la "complicidad empresarial" en crímenes internacionales, ${ }^{14}$ es decir, los directores fueron responsabilizados por usar sus posiciones dentro de la entidad para proporcionar asistencia sustancial al autor del delito a sabiendas de que participaba en la comisión de un acto ilícito. ${ }^{15}$

Uno de los legados que dejaron los juicios de Núremberg y que a día de hoy se explora para superar algunas de las fronteras delineadas, son los límites del Derecho penal internacional. En este sentido, los juicios de Núremberg establecieron que los crímenes internacionales son cometidos por personas físicas, no por entidades abstractas sin voluntad. Este principio, ha sido adoptado en los estatutos de los tribunales penales internacionales $a d h_{o c}{ }^{16}$ y de la Corte Penal Internacional

11 Estados Unidos de América contra Friedrich Flick y otros. Tribunal Militar IV, 6 (1947).

12 Estados Unidos de América contra Carl Krauch y otros. Tribunal Militar VI, 7 y 8 (1948).

13 Estados Unidos de América contra Alfred Krupp y otros. Tribunal Militar IIIA, 9 (1948).

14 Ramasastry analiza la evolución histórica de la complicidad empresarial. Según la autora, los Tribunales de Núremberg proporcionan un importante punto de partida para desarrollar la concepción moderna de la complicidad empresarial sobre la base de cooperación entre agentes económicos y Estados para cometer crímenes internacionales. RAMASASTRY, Anita. Corporate Complicity: From Nuremberg to Rangoon - An Examination of Forced Labor Cases and Their Impact on the Liability of Multinational Corporations. Berkeley Journal of International Law, v. 20, n. 1, p. 91-159 2002. 15 KALECK, Wolfgang; SAAGE-MAAß, Miriam. Corporate Accountability for Human Rights Violations Amounting to International Crimes. The Status Quo and its Challenges. Journal of international Criminal Justice, v. 8, n. 3, p. 699-724, 2010.

16 Véase, artículo 5 del Estatuto del Tribunal Penal Internacional para Ruanda, artículo 6 del Estatuto del Tribunal especial para Sierra Leona, artículo 6 del Estatuto del Tribunal Penal Internacional ad 
(CPI), por lo que las personas jurídicas no están contempladas dentro de las competencias de los tribunales penales internacionales.

Durante los trabajos preparatorios del Estatuto de Roma, se propuso la inclusión de la responsabilidad penal de las personas jurídicas dentro de las competencias de la CPI. ${ }^{17}$ Los párrafos 5 y 6 del artículo 23 propuestos por Francia en el borrador establecían que la Corte también podía ejercer jurisdicción sobre las personas jurídicas, a excepción de los Estados, cuando los crímenes se hubieran cometido en nombre de ellas o por sus agentes o representantes; y continuaba diciendo que la responsabilidad penal de las personas jurídicas no excluiría la responsabilidad penal de las personas físicas que hubieran actuado en calidad de autores o cómplices. ${ }^{18}$

La propuesta de Francia se enfocaba únicamente en las personas jurídicas de carácter privado (organizaciones criminales y empresas) para contrarrestar los efectos de ciertos comportamientos empresariales y alentar a los Estados Parte a adoptar medidas legales más severas que fueran aplicables a este tipo de entidades. Asimismo, entre los objetivos de la propuesta se encontraba aumentar las posibilidades de las víctimas de obtener una indemnización a través del régimen de reparación de la CPI.

Por otra parte, la propuesta se trataba de un modelo de responsabilidad de carácter derivado, es decir, primero se determinaba la responsabilidad penal individual, específicamente de las personas físicas con capacidad de control y de dirección que actuaran en nombre y con el consentimiento explícito de la empresa en el curso de sus actividades. ${ }^{19}$ Por tanto, al contrario de los juicios de Núremberg, la responsabilidad penal de la empresa dependía de la responsabilidad penal individual.

A pesar de que la responsabilidad penal de las personas jurídicas había experimentado cierta evolución desde su primera aproximación en los juicios de Núrem-

hoc para la antigua Yugoslavia, artículo 1 del Estatuto del Alto Tribunal Penal Iraquí, artículo 2 del Estatuto del Tribunal Especial Para el Genocidio Camboyano.

17 Véase, "Proposal Submitted by France", A/CONF.183/ C.1/L.3(16 de junio de 1998).

18 Informe del Comité Preparatorio sobre el Establecimiento de una Corte Penal Internacional, ONU A/CONF.183/2/Add.1. (14 de abril de 1998).

19 ICJ. Corporate Complicity and Legal Accountability; Report of the International Commission of Jurists Expert Legal Panel on Corporate Complicity in International Crimes, Criminal Law and International Crimes. Ginebra: ICJ, 2008. p. 56. berg y de que algunos Estados apoyaban la propuesta, ${ }^{20}$ el Estatuto de Roma no incorporó dentro de sus disposiciones este tipo de responsabilidad en las competencias de la CPI, asegurando así una solución favorable que permitiera que los Estados que se oponen filosóficamente, constitucionalmente o doctrinariamente a la responsabilidad penal de las personas jurídicas firmaran y ratificaran el Estatuto.

\subsection{La práctica actual en el Derecho penal internacional frente a los abusos cometidos en el marco de las actividades empresariales}

Aunque en la práctica internacional actual no se pueda enjuiciar a las empresas por las graves violaciones de derechos humanos que constituyen crímenes internacionales, no significa que las atrocidades que se cometen en el marco de las actividades empresariales deben quedar impunes. Las personas físicas con capacidad de control y de dirección en el seno de las empresas son sujetos capaces de activar la jurisdicción de los tribunales penales internacionales.

En este sentido, de conformidad con el artículo 25 (responsabilidad penal individual) y artículo 28 (responsabilidad de los jefes y otros superiores) del Estatuto de Roma, la CPI podrá ejercer su jurisdicción complementaria sobre los directivos de una empresa cuando sus acciones, que constituyan o contribuyan a la comisión de crímenes internacionales, se remitan al Fiscal por parte de un Estado Parte o por el Consejo de Seguridad de Naciones Unidas (artículo 13 del Estatuto de Roma).

La Físcalia ha iniciado investigaciones y enjuiciamientos en contra de directivos y ejecutivos de algunas empresas. ${ }^{21}$ Un ejemplo es el caso contra el antiguo jefe

20 CHIOMENTI, Cristina. Corporations and the International Criminal Court. En: DE SCHUTTER, Olivier (Ed.). Transnational Corporations and Human Rights. Oxford-Portland: Hart Publishing, 2006. p. 287-312.

21 Los dos fiscales que ha tenido la CPI hasta la fecha han manifestado su compromiso de investigar entidades empresariales responsables de contribuir a la comisión de crímenes internacionales. Por su parte, el fiscal Luis Moreno Ocampo en su momento hizo referencia al conflicto en la República Democrática del Congo y al papel de las empresas dedicadas a la comercialización de recursos naturales que financiaban las actividades de las fuerzas rebeldes o gubernamentales, lo que les permitirá mantener el conflicto. Mientras tanto, la fiscal Fatou Bensouda ha puesto énfasis en que las investigaciones a fondo de las finanzas que hay detrás de un conflicto ayudan a identificar posibles empresas implicadas. BATESMITH, Alex. Corporate Criminal Responsibility for War Crimes and Other Violations of International Humanitarian Law: the Impact of the 
de operaciones y periodista de la cadena de radio Kass FM, Joshua Arap Sang, acusado de crímenes de lesa humanidad por la transmisión de mensajes codificados en sus emisiones de radio que contribuyeron a la comisión de asesinatos, traslados forzosos y persecuciones ilegales en el Valle del Rift durante los sucesos violentos postelectorales que tuvieron lugar en Kenia en 2007 y 2008. Este caso fue cerrado en 2016 por falta de pruebas y testigos para mantener los cargos. ${ }^{22}$

Un caso más reciente en el que se invoca el artículo 25 del Estatuto de Roma para que la CPI investigue la participación de empresarios en crímenes internacionales es de la empresa estadounidense Chiquita Brands International, acusada de contribuir a la comisión de crímenes de lesa humanidad en Colombia mediante el pago de paramilitares y guerrilleros para proteger sus operaciones.

En mayo de 2017, de conformidad al artículo 15 del Estatuto de Roma, una coalición de OSC solicitó al Fiscal de la Corte investigar a catorce ejecutivos de la empresa. Según las OSC, los altos cargos de la empresa tenían conocimiento de las actividades criminales (homicidios, desplazamiento forzado, desaparición forzada, violencia sexual, torturas y persecuciones de civiles) del grupo paramilitar las Autodefensas Unidas de Colombia (AUC) y, a pesar de ello, supervisaron y autorizaron el pago a los bloques de las AUC. Los pagos al grupo paramilitar sirvieron para la compra de armas y municiones utilizadas en crímenes atroces cometidos en las regiones bananeras colombianas de Antioquia y Chocó. ${ }^{23} \mathrm{Al}$ momento de escribir el presente artículo la Corte no se ha pronunciado sobre el caso todavía, pero si respondiera de manera positiva a la petición de las organizaciones, lanzaría un fuerte mensaje para incidir en la conducta de los agentes corporativos.

La responsabilidad de los jefes y otros superiores

Business and Human Rights Movement. En: HARVEY, Caroline; SUMMERS, James; WHITE, Nigel D. (Ed.). Contemporary Challenges to the Laws of War: Essays in Honour of Professor Peter Rowe. Cambridge: CUP, 2014. p. 285-312.

22 Asunto Decision on Prosecutor's Application for Witness Summonses and resulting Request for State Party Cooperation [ICC-01/09-01/11-1274-Corr2], Corte Penal Internacional, 30 de abril de 2014.

23 IHCR; FIDH; COLECTIVO DE ABOGADOS JOSÉ ALVEAR RESTREPO. La contribución de ejecutivos de Chiquita en la comisión de crimenes de lesa humanidad en Colombia: Comunicación bajo el artículo 15 del Estatuto de Roma de la Corte Penal internacional. Disponible en: www.verdadabierta.com/especiales-v/2017/chiquita/InformeChiquitaBrandsCPI.pdf. también ha sido experimentada en los tribunales penales internacionales ad hoc por omisiones en los deberes de supervisión y de adoptar medidas razonables para prevenir la comisión de crímenes por parte de sus subordinados. El Tribunal Penal Internacional para Ruanda responsabilizó a Alfred Musema, director de una fábrica de té, por la participación de sus empleados en el genocidio de Ruanda. ${ }^{24}$ Los empleados utilizaron vehículos de la fábrica y la propiedad para la comisión de actos ilícitos. En este caso se clarificó la obligación de los directores y ejecutivos de adoptar las medidas necesarias para prevenir que sus subordinados cometan delitos en el desarrollo de sus actividades, especialmente cuando operan en zonas de conflicto o de inestabilidad.

A pesar de que existe la posibilidad de responsabilizar penalmente a los directivos y ejecutivos de las empresas ante una instancia intencional, se trata de una cuestión difícil de llevar a la práctica, especialmente en casos de empresas de gran tamaño debido a la dificultad de probar que las decisiones o conductas (acciones u omisiones) de los directivos y ejecutivos dieron lugar a la comisión de los crímenes. Las complejas estructuras y cadenas de suministro de las grandes empresas permiten encubrir fácilmente a los responsables individuales, difuminando cualquier conexión entre los hechos cometidos y las actuaciones de los directivos y ejecutivos de las empresas. Por lo tanto, la responsabilidad se suele trasladar al operario que realiza la acción y no a quienes la ordenan. Estas dificultades ya se veían reflejadas en los juicios de Núremberg, pues en algunos casos fue difícil probar que el acusado conocía personalmente las atrocidades cometidas en el seno de la empresa, por lo que muchos de los acusados fueron absueltos o condenados a penas leves. ${ }^{25}$

En definitiva, la responsabilidad penal de los agentes corporativos no resuelve de manera satisfactoria el problema de la participación de las empresas en crímenes internacionales que constituyen graves violaciones de los derechos humanos. Esto plantea la necesidad de establecer métodos alternativos para responsabilizar a las empresas por la falta de una organización y estructura adecuada para prevenir graves conductas ilegales en el

24 Prosecutor v. Musema, ICTR-96-13-T, Judgment, 16 November 2001, paras. 889-926 and 942-951.

25 FERENCZ, Benjamin B. An International Jurisdiction for Corporate Atrocities: Observations of a Former Nuremberg War Crimes Prosecutor. Harvard International Law Journal, v. 57, p. 15-19, 2016. 
marco de sus actividades.

En la actualidad, parte de la doctrina asume que una de las mejores vías para asegurar que los directivos y ejecutivos de las empresas se impliquen en el respeto de las normas internacionales es a través de la responsabilidad penal directa de las personas jurídicas, ya que aunque se logre identificar y sancionar a los responsables, la empresa podría continuar realizando una conducta ilícita. Por consiguiente, el enjuiciamiento directo de las empresas podría crear el elemento disuasorio necesario más allá del que supone enjuiciar a los actores individuales. En este sentido, se puede considerar la responsabilidad de las empresas mediante el modelo de imputación basado en el modelo de organización que atribuye la responsabilidad por fallas o defectos en la estructura organizacional de la persona jurídica. Este modelo ya es empleado en algunas jurisdicciones nacionales como en Australia. ${ }^{26}$

Se plantea, por tanto, la posibilidad de enmendar el Estatuto de Roma ${ }^{27}$ o de adoptar un protocolo adicional para introducir la responsabilidad penal internacional directa de las personas jurídicas dentro de las competencias ratione personae de la CPI, independientemente de la responsabilidad penal de los individuos que cometieron la conducta delictiva. ${ }^{28}$ Autores como CLAPHAM ${ }^{29}$ y JÄGERS ${ }^{30}$ presagiaban que tras la primera Conferencia de Revisión del Estatuto de Roma, que se llevó a cabo en Kampala (Uganda) en 2010, los Estados Parte incluirían la responsabilidad penal de las personas jurídicas dentro de las competencias de la CPI. Sin embargo, no se adoptó ninguna disposición relacionada con el tema,

26 IVORY, Radha; JOHN, Anna. Holding Companies Responsible? The Criminal Liability of Australian Corporations for Extraterritorial Human Rights Violations. University of New South Wales Law Journal, v. 40, n. 3, p. 1175-119, 2017.

27 KYRIAKAKIS, Joanna. Corporations before International Criminal Courts: Implications for the International Criminal Justice Project. Leiden Journal of International Law, v. 30, n. 1, p. 221-240, 2017. La extensión de la competencia de la CPI vía enmienda requerirá una mayoría de dos tercios en la reunión de la Asamblea de los Estados Parte o en una Conferencia de Revisión (artículo 121 del Estatuto de Roma).

28 Scheffer, David. Corporate Liability under the Rome Statute. Harvard International Law Journal, v. 57, p. 36-39, 2016.

29 CLAPHAM, Andrew. The Question of Jurisdiction under International Criminal Lawover Legal Persons: Lessons from the Rome Conference on an International Criminal Court. En: KAMMINGA, Menno T. y ZIA-ZARIFI, Saman (Ed.). Liability of Multinational Corporations under International Law. La Haya-Londres-Boston: Kluwer Law International, 2000. p. 139-195.

30 JÄGERS, Nicola. Corporate Human Rights Obligations: In Search of Accountability. Amberes: Intersentia, 2002. p. 220-222. ni siquiera fue retomada la propuesta de Francia. Por otra parte, también se propone reformar únicamente la Parte VII del Estatuto de Roma para determinar sanciones subsidiarias aplicables a las empresas una vez que sus órganos hayan sido declarados responsables de cometer un crimen internacional. ${ }^{31}$

La posibilidad de enmendar el Estatuto de Roma ha sido planteada por algunas delegaciones y OSC durante los períodos de sesiones del GTI, señalando que el futuro instrumento vinculante sobre empresas y derechos humanos podría corregir una omisión histórica del Derecho penal internacional. ${ }^{32}$ La propuesta de ampliación de las competencias de la CPI se basa en dos premisas: I) que las empresas se benefician de la comisión o participación en crímenes internacionales y II) que la responsabilidad penal individual, por sí sola, ha sido insuficiente hasta el día de hoy para abordar de manera adecuada una nueva realidad en la que estas entidades tienen una mayor participación en distintos ámbitos.

Entre los posibles beneficios de la extensión de las competencias de la CPI se encontrarían: el efecto disuasorio necesario para que las empresas lleven a cabo reformas en sus estructuras y operaciones por la función preventiva del Derecho penal, la garantía de una vía legal para hacer efectiva la responsabilidad de las empresas en caso de inacción por parte de los tribunales nacionales competentes $y$, finalmente, el fortalecimiento de la capacidad de los Estados para controlar las acciones de los actores corporativos. ${ }^{33}$

En suma, la falta de consenso sobre el reconocimiento de la responsabilidad penal internacional de las personas jurídicas no se debe a una limitante conceptual, sino más bien al resultado de las complejas negociaciones entre los Estados. Como señala BERNAZ, "[i]n truth, there is no conceptual reason why corporations should be immune from liability under international criminal law, and

31 WATTAD, Mohammed Said-Alden. Natural Persons, Legal Entities, and Corporate Criminal Liability under the Rome Statute. UCLA Journal of International Law and Foreign Affairs, v. 20, n. 2, p. 391-424, 2016.

32 Consejo de Derechos Humanos, Informe del segundo período de sesiones del grupo de trabajo intergubernamental de composición abierta sobre las empresas transnacionales y otras empresas con respecto a los derechos humanos, A/HRC/34/47 (4 de enero de 2017), párr. 85.

33 KYRIAKAKIS, Joanna. Corporations before International Criminal Courts: Implications for the International Criminal Justice Project. Leiden Journal of International Law, v. 30, n. 1, p. 221-240, 2017. 
the idea has never been clearly rejected." ${ }^{34}$ En otras palabras, la responsabilidad penal de las personas jurídicas no es una noción que no exista o no pueda ser concebida en el Derecho internacional, pero se requiere de un consenso político para que pueda ser incorporada expresamente en el régimen jurídico internacional.

\section{Los avances en el Derecho penal INTERNACIONAL PARA LA RESPONSABILIDAD} PENAL DE LAS EMPRESAS POR LA VULNERACIÓN DE DERECHOS HUMANOS

Algunos de los avances más destacables que han tenido lugar a nivel internacional favorecen la práctica de poner fin a la impunidad de graves abusos corporativos a través del Derecho penal internacional. Entre ellos destacan: a) el Policy Paper de la Oficina del Fiscal de la CPI sobre Priorización y Selección de Casos a ser Investigados, b) las sentencias de los casos New TV S.A.L. y Akbbar Beirut S.A.L. del Tribunal Especial para Líbano y c) el Protocolo de Malabo sobre las enmiendas al Protocolo sobre el Estatuto de la Corte Africana de Derechos Humanos y de los Pueblos. El primero de ellos mantiene el statu quo del Derecho penal internacional, enfocándose en la responsabilidad penal individual de los directivos y ejecutivos de las empresas por la comisión o complicidad en crímenes internacionales. Mientras que el segundo y el tercero ponen a prueba las fronteras del Derecho penal internacional y avanzan hacia el reconocimiento de la competencia de los tribunales internacionales para hacer efectiva la responsabilidad de las empresas por crímenes internacionales.

\subsection{El Policy Paper de la Oficina del Fiscal de la Corte Penal Internacional sobre Priorización y Selección de Casos a ser Investigados}

En septiembre de 2016, la Oficina del Fiscal de la CPI publicó, con base en el marco legal aplicable y la jurisprudencia de la Corte, un documento sobre priorización y selección de casos a ser investigados. ${ }^{35} \mathrm{El}$ Policy

34 BERNAZ, Nadia. Corporate Criminal Liability under International Law. The New TV S.A.L. and Akhbar Beirut S.A.L. Cases at the Special Tribunal for Lebanon. Journal of International Criminal Justice, v. 13, n. 2, p. 313-330, 2015.

35 Office of the Prosecutor, Policy Paper on Case Selection and Prioritisation (15 September 2016).
Paper de priorización presenta un gran potencial para que se preste mayor atención a los crímenes cometidos en el marco de las actividades empresariales.

El Policy Paper de priorización atrajo la atención de los medios internacionales y de las OSC dado que puso de manifiesto el compromiso de la Oficina del Fiscal de prestar especial consideración a la persecución de los crímenes del Estatuto de Roma que se cometan o que tengan como resultado la destrucción del medio ambiente, la explotación ilegal de los recursos naturales o el despojo ilegal de las tierras.

De acuerdo con lo señalado en el documento, estos nuevos criterios cumplen con el requisito de gravedad (escala, características, modo de comisión e impacto) para la selección de casos a ser investigados y procesados por el Fiscal, aunque no se lleva a cabo una extensión formal de los crímenes competencia de la CPI, es decir, la Oficina solo puede atender estos delitos accesorios indirectamente o, lo que es lo mismo, en la medida en que concurran elementos contextuales o materiales de crímenes que sean competencia de la Corte. No obstante, implica cierto reconocimiento de que estas situaciones de degradación ambiental son motivo de preocupación para la comunidad internacional y que en ocasiones dan pie a crímenes internacionales.

La importancia del documento no solo recae en que abre el debate sobre la posible incorporación de los atentados graves contra el medio ambiente en entornos de paz dentro de la competencia ratione materiae de la CPI, ${ }^{36}$ sino que genera un cambio en el panorama del Derecho penal internacional. ${ }^{37} \mathrm{El}$ documento eleva las expectativas de someter a un mayor escrutinio las actividades de las empresas que afectan el disfrute de los derechos humanos. Muchos de los conflictos bélicos contemporáneos tienen sus raíces en la competencia por la apropiación de los recursos naturales, ${ }^{38}$ por tanto,

36 LAMBERT, Caitlin. Environmental Destruction in Ecuador: Crimes against Humanity under the Rome Statute? Leiden Journal of International Law, v. 30, n. 3, p. 707-729, 2017.

37 GLOBAL WITNESS PRESS RELEASE. Company Executives Could Now Be Tried for Land Grabs and Environmental Destruction (15 de septiembre de 2016). Disponible en: www.globalwitness.org/en/ press-releases / company-executives-could-now-be-tried-land-grabbing-and-environmentaldestruction-historic-move-internationalcriminal-court-prosecutor/.

38 VAN DEN HERIK, Larissa; DAM-DE JONG, Daniëlla. Revitalizing the Antique War Crime of Pillage: The Potential and Pitfalls of Using International Criminal Law to Address Illegal Resource Exploitation during Armed Conflict. Criminal Law Forum, v. 22, n. 3, p. 237, 2011. 
dado los beneficios económicos y financieros en juego, la destrucción del medio ambiente, la explotación ilegal de los recursos naturales y el despojo ilegal de las tierras son acciones en las que suelen estar implicadas las empresas, especialmente las del sector extractivo o agroindustrial. ${ }^{39}$

En 2014, se solicitó a la Oficina del Fiscal la investigación de crímenes de lesa humanidad en Camboya cometidos a raíz del generalizado y sistemático acaparamiento de tierras llevado a cabo por la élite gobernante del país, ${ }^{40}$ incluidos los líderes empresariales. Las personas que viven de la tierra se oponen al acaparamiento, convirtiéndose en blancos de asesinatos, detenciones ilegales, criminalización y otros actos inhumanos. A la luz del Policy Paper, este tipo de situaciones consiguen una mayor atención del Fiscal de la CPI, que adquiere un papel más activo para hacer frente a los impactos negativos de las actividades empresariales sobre los derechos humanos.

En consecuencia, el Policy Paper puede alentar la investigación y enjuiciamiento de los directivos y ejecutivos de las empresas que sí están contemplados dentro de la jurisdicción de la CPI, lo que supone un importante avance teniendo en cuenta las limitaciones del Estatuto de Roma para hacer efectiva la responsabilidad directa de las empresas por la comisión o complicidad en crímenes internacionales.

Asimismo, como en el caso de Camboya o en el de Chiquita Brands International, el Policy Paper incita a que las OSC comuniquen de manera estratégica al Fiscal, aunque no se produzcan las investigaciones o el enjuiciamiento, potenciales casos de crímenes internacionales que se cometan o que tengan como resultado la destrucción del medio ambiente, la explotación ilegal de los recursos naturales o el despojo ilegal de las tierras a causa del desarrollo de actividades empresariales. ${ }^{41}$ Esto puede atraer la atención de los medios internacionales y generar presión sobre las empresas por los posibles impactos en su imagen y reputación.

39 BERNAZ, Nadia. An Analysis of the ICC Office of the Prosecutor's Policy Paper on Case Selection and Prioritization from the Perspective of Business and Human Rights. Journal of International Criminal Justice, v. 15, n. 3, p. 527-542, 2017.

40 Global Diligence. Communication under Article 15 of the Rome Statute of the International Criminal Court (julio de 2002). Disponible en: https://www.fidh.org/IMG/pdf/executive_summary-2.pdf.

41 FAIRLIE, Megan A. The Hidden Costs of Strategic Communications for the International Criminal Court. Texas International Law Journal, v. 51, n. 3, p. 281-319, 2016.
4.2. Las sentencias de los casos New TV S.A.L. y Akhbar Beirut S.A.L. del Tribunal Especial para Líbano

Uno de los avances, modestos pero destacables, en relación a la responsabilidad penal internacional de las empresas tuvo lugar en el Tribunal Especial para el Líbano (STL, por sus siglas en inglés), constituido para enjuiciar a los presuntos responsables de llevar a cabo el ataque del 14 de febrero de 2005 que mató a 22 personas, incluido el ex primer ministro de Líbano, Rafik Hariri, e hirió a muchos otras. Por primera vez, de manera inesperada, se determinó que un tribunal penal internacional ad hoc tenía competencia ratione personae sobre personas jurídicas por delitos contra la administración de justicia. A pesar de que se determinó en apelación la competencia del STL sobre dos casos contra empresas por desacato y obstrucción a la justicia, este precedente constituye un progreso importante en la responsabilidad penal internacional de las empresas porque pone de manifiesto dos cuestiones relevantes:

I) Que las empresas, como cualquier persona física, son susceptibles de responder por sus conductas ilícitas ante tribunales internacionales. En este sentido, era cuestión de tiempo que una conducta realizada por una empresa llamara la atención de un tribunal internacional, teniendo en cuenta que hoy en día es difícil que pasen desapercibidas en cualquier instancia legal, especialmente considerando el aumento del número de empresas y su implicación cada vez mayor en diversos ámbitos sociales, políticos, económicos, etc.

II) Que un reconocimiento expreso de la responsabilidad de las empresas facilita y agiliza los procesos penales en instancias supranacionales. La competencia del STL se determinó en apelación, lo que significa que hubo un examen y análisis previo a la cuestión. Esta cuestión puede dilatar los procesos. El reconocimiento expreso evita retrasos en el proceso y centraliza la atención, los esfuerzos y los recursos en determinar la responsabilidad de la empresa.

Debido a que la cuestión de los casos no versa sobre violaciones de derechos humanos, a continuación se analizan brevemente los antecedentes y las cuestiones más destacables de las conclusiones del Panel de Apelaciones respecto a la responsabilidad de las empresas, particularmente en el caso contra New TV S.A.L.

En 2014, el Juez David Baragwanath del STL emitió 
órdenes contra personas físicas y empresas de comunicación. Los cargos se referían a la publicación en un programa de televisión y en un periódico de los nombres de los supuestos testigos confidenciales en el proceso ante el STL. Según el Juez Baragwanath, la publicación de los nombres de los testigos constituía una injerencia deliberada en la administración de justicia. El Juez Nicola Lettieri fue designado para resolver los casos contra Karma Mohamed Tahsin al-Khayat de Al-jadeed TV y la empresa matriz de la emisora New TV S.A.L., ${ }^{42}$ y contra Ibrahim Mohamed Al-Amin de Al-Akbbar y la empresa matriz del periódico Akbbar Beirut S.A.L., acusados de desacato y obstrucción a la justicia de acuerdo a la Regla 60 bis de las Reglas de Procedimiento y Prueba del Tribunal. ${ }^{43}$

En julio de 2014, el Juez Lettieri falló a favor de la defensa de New TV S.A.L. y Karma Khayat, que impugnaron la competencia del STL debido a que no existía una base legal en el Estatuto del Tribunal, en las Reglas de Procedimiento y Prueba o en el Derecho penal internacional para iniciar un proceso contra una persona jurídica. ${ }^{44} \mathrm{El} \mathrm{Juez,} \mathrm{por} \mathrm{tanto,} \mathrm{determinó} \mathrm{que} \mathrm{la} \mathrm{Regla} 60$ bis se aplicaba solamente a las personas físicas. ${ }^{45}$ No obstante, en ese mismo año el Panel de Apelación revirtió la decisión del Juez Lettieri, determinando que es parte del interés de la justicia interpretar que la competencia personal del Tribunal engloba a las personas jurídicas de

42 Asunto Ms Karma Mohamed Tahsin Al Khayat and Al Jadeed (STL-14-05), Tribunal Especial para el Líbano, 31 de enero de 2014. 43 Asunto Mr Ibrahim Mohamed Ali Al Amin and Akhbar Beirut S.A.L. (STL-14-06), Tribunal Especial para el Líbano, 31 de enero de 2014.

44 En el caso contra la empresa Akbbar Beirut S.A.L. se impugnó la competencia del STL del mismo modo que en el caso contra New TV S.A.L. A pesar de que el Panel de Apelación ya había resuelto en el caso New TV S.A.L. que el STL era competente sobre las personas jurídicas en los casos de desacato y obstrucción de la justicia, el Juez Lettieri rechazó una vez más el caso debido a la falta de competencia sobre personas jurídicas. De acuerdo con la opinión del Juez, la decisión en New TV S.A.L. era un caso único y no se apoya en la jurisprudencia internacional, por lo que la decisión del Panel de Apelación era solo aplicable a ese caso. Asimismo, se apoyó en que uno de los jueces del Panel discrepó por lo que la decisión no fue unánime, y por lo tanto lleva menos autoridad que una decisión unánime. Véase, Asunto Decision on Motion challenging jurisdiction Mr Ibrahim Mohamed Ali Al Amin and Akhbar Beirut S.A.L. (STL-14-06/PT/CJ), Tribunal Especial para el Líbano, 6 de noviembre de 2014.

45 Decision on Motion challenging jurisdiction and on request for leave to amend order in lieu of an indictment, New TV S.A.L. and Khayat (STL-14-05/PT/CJ), Tribunal Especial para el Líbano, 24 de Julio de 2014. conformidad con la Regla 60 bis. ${ }^{46}$ Pocos meses después el Panel de Apelación resolvió en el mismo sentido en el caso Akhbar Beirut S. A.L.

Estos casos constituyen un precedente de gran relevancia hacia la responsabilidad penal internacional de las empresas pues, por primera vez, aunque de manera simbólica y con un alcance limitado, un tribunal penal internacional responsabilizó a dos empresas, desafiando así el enfoque individualista del Derecho penal internacional. Esta decisión, sin duda, podría utilizarse en el futuro para un caso sobre empresas y derechos humanos. Asimismo, podría servir de referencia en otras instancias internacionales como prueba de que las empresas pueden cometer delitos con incidencia en el ámbito internacional y pueden ser procesadas por ello. Algunos autores, como BERNAZ, esperan que estos precedentes no queden aislados y que otros tribunales penales internacionales, incluidos los tribunales nacionales, comiencen a considerar la responsabilidad penal de las empresas en virtud del Derecho internacional. ${ }^{47}$

\subsection{El Protocolo de Malabo sobre las enmiendas al Protocolo sobre el Estatuto de la Corte Africana de Derechos Humanos y de los Pueblos}

La Unión Africana adoptó en 2014 el Protocolo de Malabo sobre las enmiendas al Protocolo sobre el Estatuto de la Corte Africana de Derechos Humanos y de los Pueblos, que entrará en vigor una vez sea ratificado por 17 Estados miembros de la Unión Africana (artículo 11). El Protocolo crea una nueva sección de Derecho penal internacional que atribuye a la Corte Africana la competencia para conocer de casos de crímenes internacionales cometidos por empresas operando en Áfri$\mathrm{ca}^{48}$

46 Del mismo modo, el Panel de Apelación, por razones de seguridad jurídica y para evitar la fragmentación de la ley, resolvió la impugnación de competencia en el caso Akbbar Beirut S.A.L., señalando que el STL sí era competente para conocer de los casos contra las personas jurídicas por desacato y obstrucción de la justicia. Véase, Decision on Interlocutory Appeal Concerning Personal Jurisdiction in Contempt Proceedings, Akhbar Beirut S.A.L. and Ibrahim Mohamed Al-Amin (STL-14-06/PT/AP/AR126.1), Tribunal Especial para el Líbano, 23 de enero de 2015.

47 BERNAZ, Nadia. Corporate Criminal Liability under International Law. The New TV S.A.L. and Akhbar Beirut S.A.L. Cases at the Special Tribunal for Lebanon. Journal of International Criminal Justice, v. 13, n. 2, p. 313-330, 2015.

48 El artículo 46C sobre responsabilidad penal empresarial del Protocolo de Malabo se lee de la siguiente manera: "1. For the purpose of this Statute, the Court shall have jurisdiction over legal persons, with 
El Protocolo de Malabo es el primer instrumento estatutario que incorpora la responsabilidad penal directa de las personas jurídicas mediante el modelo de imputación basado en el modelo de organización. En lugar de centrarse en el comportamiento de determinados individuos en el seno de la empresa, la responsabilidad se deriva de la implementación de sus políticas formales e informales y del conocimiento de la comisión del delito (artículo 46C.2-5).

Esto demuestra que está a la vanguardia de los modelos de imputación de responsabilidad penal de las personas jurídicas, pues mediante este modelo se busca incentivar cambios en las prácticas y en las políticas de las empresas. Por otra parte, la responsabilidad penal de las personas jurídicas no excluye la responsabilidad de las personas físicas que cometieron o fueron cómplices del crimen (artículo 46C.6), lo que evita que los miembros individuales se escuden en la estructura de las empresas para evadir su responsabilidad.

Se pueden destacar tres cuestiones relevantes en relación al Protocolo de Malabo. En primer lugar, si se adoptaran las enmiendas, se constituiría el primer tribunal internacional que reconocería expresamente su competencia sobre las empresas. En segundo lugar, se trata de una propuesta que surge de los Estados del Sur Global para poner fin a la impunidad corporativa. Esto reafirma el hecho de que las actividades de las empresas tienen un mayor impacto negativo en el Sur Global, como en el continente africano, donde se documentan múltiples conflictos relacionados con la extracción de los recursos naturales por parte de grandes empresas del sector minero en países como Angola, Sierra Leona, Liberia y la República Democrática del Congo (RDC). ${ }^{49}$

the exception of States. 2. Corporate intention to commit an offence may be established by proof that it was the policy of the corporation to do the act which constituted the offence. 3. A policy may be attributed to a corporation where it provides the most reasonable explanation of the conduct of that corporation. 4. Corporate knowledge of the commission of an offence may be established by proof that the actual or constructive knowledge of the relevant information was possessed within the corporation. 5. Knowledge may be possessed within a corporation even though the relevant information is divided between corporate personnel. 6. The criminal responsibility of legal persons shall not exclude the criminal responsibility of natural persons who are perpetrators or accomplices in the same crimes." Sobre el Protocolo de Malabo, véase AMNISTÍA INTERNACIONAL. Malabo Protocol: Legal and Institutional Implications of the Merged and Expanded African Court. Londres: Amnesty International, 2016.

49 Final Report of the Panel of Experts on the Illegal Exploitation of Natural Resources and Other Forms of Wealth of the Democratic Republic of the Congo, ONU S/2002/1146 (16 de octubre de 2002); Final report of the Monitoring Mechanism on Angola
Esta propuesta supone una respuesta a las demandas de la sociedad civil para establecer vías legales que creen un contrapeso a las actuaciones de las empresas transnacionales en los Estados africanos incapaces o indispuestos a responsabilizar a las empresas que operan en sus territorios.

Finalmente, el Protocolo de Malabo no solo concedería a la Corte Africana competencia sobre los crímenes internacionales contemplados en el Estatuto de Roma, sino también sobre otro tipo de conductas como corrupción, tráfico de personas, tráfico de drogas, tráfico de residuos peligrosos y explotación ilícita de recursos naturales, entre otros (artículo 28A.1). Tras probarse el alcance y la viabilidad del enjuiciamiento de las empresas en esta instancia internacional, se podría implementar en la CPI y en otros tribunales penales internacionales ad hoc.

\section{LA RESPONSABILIDAD PENAL DE LAS EMPRESAS A NIVEL NACIONAL POR LA VULNERACIÓN DE DERECHOS HUMANOS}

A la luz de lo señalado anteriormente y de los obstáculos y límites descritos en el Derecho penal internacional para hacer efectiva la responsabilidad de las empresas, en este apartado se examina la viabilidad de responsabilizar penalmente a las empresas ante los tribunales penales a nivel nacional por las vulneraciones de derechos humanos cometidas en el marco de sus actividades.

El vacío jurídico del Derecho penal internacional en relación a las empresas se ve subsanado parcialmente a nivel nacional, ya que, como se ha indicado, si bien la responsabilidad penal de las empresas aún no es aceptada universalmente en todas las jurisdicciones, es cierto que cada vez son más los Estados que reconocen la responsabilidad penal de las personas jurídicas, como responsables directos o como cómplices de actos ilícitos.

No obstante, existen diferentes posturas en los ordenamientos jurídicos internos respecto a esta cuestión. Por ejemplo, de acuerdo con un estudio comparativo

Sanctions, S/AC.31/2000/CRP.1 (21 de diciembre de 2000); Report of the Panel of Experts appointed pursuant to Security Council Resolution 1306 (2000), paragraph 19, in relation to Sierra Leone, S/2000/1195 (20 de diciembre de 2000). 
encargado por la Unión Europea (UE) en relación con la responsabilidad penal de las personas jurídicas, el 50 por ciento de los Estados miembros de la UE han introducido la responsabilidad penal general en sus sistemas jurídicos, de los cuales, el 41 por ciento reconoce la responsabilidad penal de las personas jurídicas solo para delitos específicos. El resto reconoce la responsabilidad administrativa de las empresas por la comisión de delitos. ${ }^{50}$

A su vez, un gran número de Estados han incorporado dentro de sus ordenamientos penales los crímenes internacionales contemplados en el Estatuto de Roma. ${ }^{51}$ No solo los Estados Parte del Estatuto de Roma, sino también algunos que no lo son, como Japón, India o los Estados Unidos, que han incorporado uno o más crímenes dentro de su legislación nacional. ${ }^{52}$

Asimismo, los Estados, en cumplimiento con sus obligaciones y compromisos internacionales, incorporan y definen sanciones penales en sus ordenamientos internos derivados de tratados internacionales de derechos humanos y de derecho humanitario, muchos de los cuales también fomentan la responsabilidad penal de las empresas a nivel nacional. ${ }^{53}$ Es decir, algunos instrumentos internacionales contienen disposiciones para que los Estados Parte adopten las medidas legales aplicables a las personas jurídicas que cometan los hechos ilícitos en determinados ámbitos expuestos a la delincuencia empresarial. Por ejemplo, el Protocolo facultativo de la Convención sobre los Derechos del Niño relativo a la Venta de Niños, la Prostitución Infantil y la utilización de Niños en la Pornografía de 2000 señala que los Estados Parte adoptarán, cuando proceda, las disposiciones que permitan hacer efectiva la responsabilidad de las personas jurídicas, que podrá ser penal, civil o administrativa (artículo 3.4). ${ }^{54}$ Esta fór-

50 VERMEULEN, Gert; DE BONDT, Wendy; RYCKMAN, Charlotte. Liability of legal persons for offences in the EU. Amberes: Maklu, 2012. p. 79.

51 RAMASASTRY, Anita; THOMPSON, Robert C. Commerce, Crime and Confict Legal Remedies for Private Sector Liability for Grave Breaches of International Law. A Survey of Sixteen Countries New Security Programme Economic Agendas and Civil Wars. Executive Summary. Oslo: FAFO, 2006. p. 16-17.

52 DE JONGE, Alice. Transnational corporations and international law. Accountability in the global business environment. CheltenhamNorthampton: Edward Elgar, 2001. p. 139.

53 MONGELARD, Éric. Responsabilidad civil de las empresas por violaciones del derecho internacional humanitario. International Review of the Red Cross, n. 863, p. 1-30, 2006.

54 Otros instrumentos internacionales que contemplan la respon- mula discrecional de dejar elegir a los Estados el tipo de responsabilidad se replica en la mayoría de los tratados. De hecho, esta disposición se contempla en el Proyecto de artículos sobre crímenes contra la humanidad de la Comisión de Derecho Internacional que establece la responsabilidad de las personas jurídicas está sujeta a los principios jurídicos de cada Estado.

Lo anterior indica que en varias jurisdicciones existe un riesgo latente para las empresas de ser responsables penalmente por graves violaciones de derechos humanos. En Europa se registran un número considerable, pero limitado, de procesos penales por violaciones de derechos humanos cometidas en el marco de las actividades de las empresas en terceros Estados. ${ }^{55}$ La mayoría de estos casos están relacionados con un comportamiento negligente de las empresas matrices por no prevenir dichos abusos. Sin embargo, la práctica de responsabilizar directamente a las empresas es limitada, por lo que en la mayoría de casos que se han llevado por la vía penal se intenta responsabilizar a los directivos o ejecutivos como vía alternativa para modificar el comportamiento de las empresas.

En Alemania, por ejemplo, se presentó una denuncia en la oficina del fiscal de Frankfurt am Main contra dos ejecutivos de la empresa Labmeyer International $\mathrm{GmbH}$, responsable de la construcción de la represa de Merowe en el norte de Sudán. Se les acusó de provocar la inundación de más de 30 aldeas, el desplazamiento de la población y la destrucción de sus medios de subsistencia. ${ }^{56}$ Asimismo, varias OSC denunciaron en Tübingen al director de la empresa Danzer Group por su complicidad en delitos de abusos sexuales, detenciones ilegales, incendios provocados y graves daños cometidos por las fuerzas de seguridad congolesas durante un

sabilidad penal de las personas son: la Convención Internacional sobre la Represión y el Castigo del Crimen de Apartheid, de 30 de noviembre de 1973 (artículo 2.f); el Convenio Internacional para la Represión de la Financiación del Terrorismo, de 9 de diciembre de 1999 (artículo 5); y, la Convención de las Naciones Unidas contra la Delincuencia Organizada Transnacional, de 15 de noviembre de 2000 (artículo 10).

55 ENNEKING, Liesbeth et al. Zorgplicbten van nederlandse ondernemingen inzake internationaal maatschappelijk verantwoord ondernemen. Een rechtsvergelijkend en empirisch onderzoek naar de stand van het Nederlandse recht in het licht van de UN Guiding Principles. Ámsterdam: WODC, 2015.

56 ECCHR. Reckless development: forced displacement due to Lahmeyer dam construction (mayo, 2016). Disponible en: www.ecchr. eu/en/our_work/business-and-human-rights/lahmeyer-case.html. 
ataque a la población de Bongulu. ${ }^{57}$ En los Países Bajos han tenido lugar los conocidos casos contra los empresarios holandeses, Guus Kouwenhoven y Frans van Anraat, que fueron condenados por facilitar la comisión de crímenes internacionales al exportar armas y productos químicos a Liberia e Iraq, respectivamente..$^{58}$

$\mathrm{Si}$ bien los casos anteriores son importantes en el ámbito de empresas y derechos humanos, su contenido es escaso en relación a la responsabilidad de las empresas per se como personas jurídicas. No obstante, existen otros casos que constituyen un referente importante que apuntan en la dirección correcta para fomentar el uso de la vía penal como un mecanismo judicial para hacer efectiva la responsabilidad de las empresas por la comisión o complicidad en graves violaciones de derechos humanos. Especialmente, representan una evidencia de que algunos conceptos del derecho interno de los Estados se pueden trasladar de manera cautelosa al ámbito internacional para hacer frente a la impunidad corporativa.

Por ejemplo, en Suiza, en 2013, se abrió una investigación penal en contra de la empresa minera Argor-Heraeus $S A$, acusada de blanqueo de capitales y pillaje de oro saqueado por un grupo armado ("Front nationaliste et intégrationniste") del noreste de la RDC. Los beneficios obtenidos de la venta de oro por los grupos armados sirven para financiar sus operaciones y para la compra de armas. Se alegaba la responsabilidad penal de la empresa por un delito contemplado en la legislación suiza que se deriva de los instrumentos internacionales aplicables a los conflictos armados no internacionales. ${ }^{59}$

En el mismo año, en los Países Bajos, la empresa Lima Holding B.V. fue acusada por varias OSC de complicidad en violaciones del Derecho internacional humanitario cometidas en los territorios palestinos ocupados por Israel, mediante el suministro de grúas y

57 GLOBAL WITNESS. Criminal Complaint against Senior Manager of Danzer Accountability for Human Rights Violations in the Democratic Republic of Congo. Disponible en: www.globalwitness.org/en/archive/ criminal-complaint-accuses-senior-manager-danzer-group-responsibility-over-human-rights/.

58 Public Prosecutor v. Guus Kouwenhoven, Tribunal Supremo de los Países Bajos [ECLI:NL:HR:2010:BK8132], 20 de abril 2010; Public Prosecutor v. van Anraat, Tribunal de Distrito de La Haya [LJN AU8685], 23 de diciembre de 2005.

59 BUSINESS \& HUMAN RIGHTS RESOURCE CENTRE. Argor-Heraeus investigation (re Dem. Rep. of Congo). Disponible en: https://business-humanrights.org/en/argor-heraeus-investigationre-dem-rep-of-congo. otros servicios para la construcción del muro. En 2017, se presentó una denuncia penal ante el fiscal holandés en contra del banco Rabobank por el blanqueó de las ganancias de los cárteles de droga mexicanos y por facilitar la comisión de crímenes de lesa humanidad y la tortura de civiles mexicanos a manos de estos cárteles. ${ }^{60}$

En Francia, las empresas Amesys ${ }^{61}$ y Q smos $^{62}$ están bajo investigación por suministrar respectivamente equipos de vigilancia al gobierno de Libia y Siria, que fueron utilizados para llevar a cabo detenciones arbitrarias, torturas y tratos inhumanos y degradantes a los opositores de dichos gobiernos. En 2016, antiguos empleados de la cementera Lafarge junto con organizaciones de la sociedad civil interpusieron una denuncia penal contra la empresa por la complicidad de crímenes de guerra y de lesa humanidad cometidas a través de las actividades de su filial en Siria entre 2013 y 2014. Según las organizaciones de la sociedad civil, la empresa pagaba a grupos armados en Siria, incluido al Estado Islámico (ISIS), para proteger sus actividades, violando así las sanciones impuestas por organismos internacionales y contribuyendo financieramente al conflicto armado en la región. En 2018, meses después de que los directivos de la empresa estuvieran bajo investigación formal por el financiamiento del terrorismo, la empresa Lafarge fue imputada por los jueces de instrucción por complicidad en crímenes de lesa humanidad, financiamiento de una empresa terrorista y por poner en peligro la vida de las personas. Además, se le ordenó un depósito de seguridad antes del juicio. ${ }^{63}$

En vista de los casos referidos en este apartado, se puede afirmar que la vía penal a nivel nacional presenta importantes posibilidades para responsabilizar a las

60 D'OLIVEIRA, Prakken. Aangifte tegen Rabobank Groep vanwege witwassen van winsten van Mexicaanse drugskeartels. Disponible en: http:// www.prakkendoliveira. $\mathrm{nl} / \mathrm{nl} /$ nieuws/aangifte-tegen-rabobankgroep-vanwege-witwassen-van-winsten-van-mexicaanse-drugskartels/.

61 SHERPA. 2006 Annual Report (2 de mayo de 2007). Disponible en: www.asso-sherpa.org/wp-content/uploads/2013/09/Annualreport-2006.pdf.

62 BUSINESS \& HUMAN RIGHTS RESOURCE CENTRE. Qosmos investigation (re Syria). Disponible en: https://business-humanrights.org/en/qosmos-investigation-re-syria.

63 ECCHR. French Judiciary indicts former directors of Lafarge in Syria case. Disponible en: www.ecchr.eu/en/business-and-human-rights/ lafarge-syria.html; ECHHR. Landmark Decision: Company Lafarge Indicted - Complicity in Crimes Against Humanity Included. Disponible en: https://www.ecchr.eu/fileadmin/Pressemitteilungen_ englisch/PR_Lafarge_Syria_Indictment_CrimesHumanity_Sherpa_ECCHR_20180628.pdf. 
empresas, o bien a sus directivos y ejecutivos, por la comisión o complicidad en graves violaciones de derechos humanos. No obstante, la investigación y enjuiciamiento penal de estos casos están ligados a una serie de obstáculos prácticos, legales e incluso políticos que reducen el potencial de esta vía, entre ellos: a) la competencia internacional en materia penal, b) el inicio de la investigación y del proceso penal y c) la falta de cooperación judicial en la investigación, que se analizan con mayor detalle a continuación.

\subsection{La competencia internacional en materia penal}

Las empresas transnacionales realizan sus actividades en varios países, lo que equivale a una pluralidad de escenarios donde pueden cometer actos ilícitos. Por tanto, las cuestiones competenciales también juegan un papel importante en la investigación y enjuiciamiento penal de las empresas ya que, por lo general, se trata de casos de carácter extraterritorial que se cometen en el marco de sus operaciones globales o de sus cadenas de suministro. Así surge la cuestión de qué tribunal es competente cuando se sospecha o se denuncia que un directivo o una empresa registrada o domiciliada en una jurisdicción (bome State) comete o contribuye a la comisión de un delito en otra (bost State).

La jurisdicción penal tiene primordialmente un carácter territorial y, por tanto, se suele ejercer sobre los hechos y empresas locales donde tuvieron lugar los actos delictivos (bost State). Esto, junto con las doctrinas de personalidad jurídica separada y de responsabilidad limitada de las entidades que conforman un grupo corporativo, escudan a las empresas matrices domiciliadas en los home States de cualquier riesgo de responsabilidad asociado a sus actividades globales.

No obstante, casi todos los Estados de conformidad a los principios reconocidos por el Derecho internacional ejercen y reconocen de forma unilateral la jurisdicción penal extraterritorial para el enjuiciamiento y sanción de los delitos más graves cometidos más allá de sus fronteras nacionales. En este sentido, la Corte Internacional de Justicia en el caso Lotus señaló que "in all systems of law the principle of the territorial character of criminal law is fundamental, it is equally true that all or nearly all these systems of law extend their action to offences committed outside the territory of the State which adopts them, and they do so in ways which vary from State to State." ${ }^{94}$

Por lo general, para investigar y enjuiciar los delitos con elementos de extranjería sin afectar la soberanía y los intereses legítimos de otros Estados se basan en los principios de territorialidad, de personalidad y de jurisdicción universal. ${ }^{65}$ La mayoría de los casos de empresas y derechos humanos llevados por la vía penal se basan en el principio de personalidad ya que la responsabilidad penal de los nacionales de un Estado por actos cometidos en terceros Estados no plantea, en principio, problemas competenciales. Por ejemplo, el caso de Lima Holding B.V. y los de Amesys y Qosmos se apoyaron en dicho principio ya que las empresas estaban constituidas en la jurisdicción de los Estados, respectivamente.

Por su parte, el principio de territorialidad también permite a los tribunales penales de un Estado conocer los actos delictivos en terceros Estados cuando una empresa no adopta las medidas necesarias para prevenir los delitos o, en su defecto, participa mediante órdenes, ejerciendo una influencia o facilitando la comisión. Sin embargo, la jurisdicción universal no presenta las mismas oportunidades a pesar de que no requiere vínculos territoriales o nacionales, ya que solo se activa con un número limitado de crímenes contemplados en el Derecho internacional y, por lo general, requiere la presencia del acusado dentro de la jurisdicción del Estado.

\subsection{El inicio de la investigación y del proceso penal}

A pesar de que los tribunales puedan ser competentes para conocer de actos delictivos en terceros Estados, pocas veces comienzan las acciones legales necesarias para investigar o enjuiciar a sus empresas o a sus filiales por delitos cometidos en el extranjero, incluso si su ordenamiento jurídico cuenta con los recursos y las bases legales para ejercer la jurisdicción penal extraterritorial o se tienen los conocimientos y las pruebas necesarias. ${ }^{66}$

64 Sentencia del Caso Lotus Francia v. Turquía [CPJI, serie A, núm. 10], Corte Internacional de Justicia, 7 de septiembre de 1927, párr. 50.

65 ZERK, Jennifer A. Towards a fairer and more effective system of domestic law remedies. A report prepared for the Office of the UN High Commissioner for Human Rights. Disponible en: http://www. ohchr.org/Documents/Issues/Business/DomesticLawRemedies/ StudyDomesticeLawRemedies.pdf. p. 42-43.

66 SKINNER, Gwynne; MCCORQUODALE, Robert; DE SCHUTTER, Olivier. The Third Pillar. Access to Judicial Remedies for Human Rights Violations by Transnational Business. Bruselas- 
Los tribunales penales han demostrado ser extremadamente selectivos en los casos que se abren a proceso. Así, en la mayoría de las jurisdicciones existe una autoridad judicial con la facultad discrecional u obligatoria de ejercer la acción pública en todos los procesos penales no reservados a denuncias privadas. En algunas ocasiones las decisiones de estas autoridades son inapelables. En otros casos también los afectados pueden interponer querellas, sin embargo, le corresponde a la autoridad competente decidir si se abre una investigación o el proceso.

Las razones más comunes por las que no suele abrirse la investigación y el proceso se deben a la prioridad que un tribunal le da a los casos con hechos o víctimas que se encuentran en un tercer Estado en relación a las situaciones que tienen lugar dentro de su jurisdicción territorial. Es razonable (de cierto modo) que la prioridad recaiga en los casos dentro de su competencia territorial por el acceso directo a las víctimas y a la evidencia para obtener una posible condena. Por el contrario, los casos extraterritoriales, sin tener la certeza de ejecutar una posible sentencia, requieren de conocimientos especializados y recursos adicionales para llevar a cabo la investigación o el proceso. Por tanto, los Estados no suelen priorizar los delitos corporativos que tienen lugar en terceros Estados ya que las empresas implicadas se ubican en múltiples jurisdicciones y, por tanto, las investigaciones para determinar la responsabilidad de una empresa por delitos cometidos en el extranjero pueden parecer particularmente desalentadoras.

Por otra parte, los casos contra las empresas a menudo atraen presiones externas que limitan las actuaciones de los órganos judiciales por los costes políticos y económicos, es decir, por los posibles conflictos de intereses, lo que puede crear interferencias políticas que lleven a que la autoridad competente decida no dar inicio a la investigación o al proceso penal por motivos políticos en lugar de razones de justicia. A pesar del carácter independiente de las autoridades judiciales, existe una gran presión externa del poder ejecutivo cuando están en juego intereses políticos y económicos, lo que afecta la decisión de ejercer o no la acción penal o, luego de ejercitarla, no mantenerla. Esto se debe a los efectos negativos en las relaciones existentes con el sector privado $\mathrm{o}$, incluso, entre el Estado en el que se han cometido los delitos y el Estado que pretende ejercer su jurisdicción penal extraterritorial.

Londres: ICAR-CORE-ECCJ, 2013. p. 32-33.
En el caso de Lima Holding B.V. el fiscal holandés se negó a seguir el caso argumentando que la contribución de la empresa en la construcción del muro fue menor y que la complejidad del caso requería una importante cantidad de recursos para llevar a cabo la investigación. En la misma línea, el caso contra la empresa Argor-Heraeus en Suiza se cerró en 2015 por falta de acceso a las pruebas.

\subsection{La cooperación y la asistencia judicial en la investigación}

Dada la complejidad y el carácter extraterritorial de los casos, se requiere que los Estados empleen recursos humanos y económicos para acceder a las víctimas, a los testigos y a las evidencias que prueben los elementos constitutivos del delito. En este sentido, algunas jurisdicciones como Alemania, Bélgica, Dinamarca, Noruega, los Países Bajos, el Reino Unido y Suecia cuentan con unidades especiales dedicadas a la investigación de crímenes de guerra, de lesa humanidad y crímenes internacionales, incluyendo los cometidos en el extranjero. ${ }^{67}$

El Grupo de Trabajo de las Naciones Unidas sobre empresas y derechos humanos ha recomendado la creación de unidades especializadas en la investigación y el enjuiciamiento que cuenten con expertos en las causas transfronterizas de empresas y derechos humanos. Si ya existen unidades dedicadas a los crímenes de guerra o de otro tipo, los Estados deben alentar a que estas también aborden las causas de derechos humanos relacionadas con actividades empresariales y las causas en las que intervengan infractores individuales. De hecho, la Dependencia Especializada en Crímenes Internacionales de los Países Bajos se hizo cargo del caso van Anraat y recabó pruebas procedentes de diversos países, entre otros, Bélgica y los Estados Unidos. ${ }^{68}$ Asimismo, la Unidad Especializada en Crímenes de Guerra y Crímenes de Lesa Humanidad del Tribunal de Grande Instance de Pa-

67 ZERK, Jennifer A. Towards a fairer and more effective system of domestic law remedies. A report prepared for the Office of the UN High Commissioner for Human Rights. Disponible en: http://www. ohchr.org/Documents/Issues/Business/DomesticLawRemedies/ StudyDomesticeLawRemedies.pdf.

68 Consejo de Derechos Humanos, Mejores prácticas y forma de aumentar la eficacia de la cooperación transfronteriza entre los Estados en lo que respecta a la aplicación de la legislación en la cuestión de las empresas y los derechos humanos: Estudio del Grupo de Trabajo sobre la cuestión de los derechos humanos y las empresas transnacionales y otras empresas, A/HRC/35/33 (25 de abril de 2017), párr. 24 y 94. 
rís está a cargo de las investigaciones en los casos contra las empresas Amesys, Qosmos y Lafarge.

En los casos que tratan delitos cometidos en el seno de grupos corporativos, no solo se requiere de unidades especializadas o recursos necesarios para investigar este tipo de ilícitos extraterritoriales, sino que es imprescindible la cooperación y la asistencia judicial entre los Estados, especialmente para que las autoridades del home State tengan acceso a la información y a las evidencias localizadas en el host State. La investigación en los casos de empresas y derechos humanos, por tanto, debe superar las diferencias culturales, las distancias geográficas y temporales y las circunstancias sociales en el lugar de los hechos para reunir las evidencias suficientes para llevar a juicio a la presunta empresa responsable. ${ }^{69}$

Por consiguiente, se requiere del permiso de otros Estados para realizar las diligencias judiciales del proceso penal (notificación, una diligencia de prueba, un acto jurídico o una medida cautelar). Es decir, las autoridades encargadas de la investigación no pueden realizarla en un tercer Estado sin la autorización o el consentimiento de sus homólogos pues se consideraría una violación de la integridad territorial y a su soberanía. No obstante, la cooperación y asistencia judicial no siempre garantizan de manera ágil y eficiente las diligencias judiciales del proceso penal, ya que las autoridades locales del lugar donde se cometieron los actos ilícitos no siempre están dispuestas a cooperar, a pesar de haber un tratado de cooperación vigente. En el caso de Lima Holding B.V. el fiscal holandés observó también que, en términos prácticos, sería difícil seguir adelante con el caso debido a la posible falta de cooperación de las autoridades israelíes en la cuestión en concreto.

En el ámbito de la cooperación y la asistencia judicial recíproca entre Estados, existen varios instrumentos de alcance regional e internacional, que constituyen, en el mejor de los casos, un sistema irregular de normas que hasta ahora no han permitido la cooperación eficiente

69 El informe del ACNUDH sobre el acceso a los recursos judiciales contiene una serie de recomendaciones (objetivos 9-10) para mejorar la cooperación judicial entre Estados para garantizar el acceso a la justicia a las víctimas de abusos de derechos humanos por empresas. Los objetivos de estas recomendaciones son facilitar y agilizar la detección, investigación, persecución y la represión para este tipo de casos específicos. Véase, Mejorar la rendición de cuentas y el acceso a las reparaciones para las víctimas de violaciones de los derechos humanos relacionadas con actividades empresariales, $10 \mathrm{de}$ mayo de 2016, Doc. ONU. A/HRC/32/19. en todos los ámbitos. ${ }^{70}$ En las sesiones del GTI se resaltó que la cooperación entre los Estados y los órganos judiciales era fundamental ya que con frecuencia el home State y el host State no cooperan en las investigaciones ni el reconocimiento mutuo de las sentencias de los tribunales nacionales.

Así, el instrumento vinculante se presta como una herramienta para fortalecer y mejorar la cooperación en los casos extraterritoriales, por lo que se ha sugerido que se reafirmen las obligaciones de los Estados de concertar acuerdos bilaterales y multilaterales para facilitar las solicitudes de asistencia letrada y la realización de investigaciones transfronterizas; el establecimiento de mecanismos de intercambio de información; y, finalmente, llevar a cabo actividades adecuadas de capacitación, información y apoyo en materia de cumplimiento de la ley. ${ }^{71}$

\section{Conclusiones}

El Derecho penal y la justicia penal se constituyen como una de las vías judiciales para hacer efectiva la responsabilidad de las empresas por graves violaciones de derechos humanos. Este mecanismo judicial se alinea a los objetivos del primer y tercer pilar de los Principios Rectores, por lo que merece un espacio en los Planes de Acción Nacional sobre empresas y derechos humanos como un mecanismo a ser reforzado para garantizar a las víctimas de abusos corporativos el acceso a la justicia.

Los casos llevados por la vía penal no solo alertan a las empresas de que sus acciones están sujetas a un mayor escrutinio, sino que también proporcionan formas innovadoras de garantizar justicia a las víctimas de abusos corporativos. En algunas jurisdicciones de Derecho continental, las víctimas incluso pueden obtener una indemnización civil del proceso penal. Asimismo, a diferencia de la vía civil y de la administrativa, la responsabilidad penal implica una mayor condena moral.

A pesar del potencial del Derecho penal como me-

70 ICJ. Proposals for Elements of a Legally Binding Instrument on Transnational Corporations and Other Business Enterprises. Ginebra: ICJ, 2016. p. 32.

71 Consejo de Derechos Humanos, Informe del segundo período de sesiones del grupo de trabajo intergubernamental de composición abierta sobre las empresas transnacionales y otras empresas con respecto a los derechos humanos, A/HRC/34/47 (4 de enero de 2017), párr. 110. 
canismo para garantizar justicia a los afectados, son (relativamente) pocos los casos de empresas y derechos humanos que se han llevado a través de esta vía. Aún existen diversos obstáculos tanto doctrinales como procesales, políticos y prácticos que merecen especial atención para explotar el potencial de esta vía judicial.

La inclusión de las personas legales dentro de la competencia de la CPI es un tema pendiente en la agenda internacional. Los avances descritos en el presente artículo en el Derecho penal internacional no solo representan un avance en la dirección correcta para hacer frente a la impunidad de los graves abusos cometidos en el marco de las actividades de las empresas, sino que son una muestra de la falta de voluntad política de los Estados para reconocer la responsabilidad penal internacional de las personas jurídicas. La experiencia de los Estados respecto a esta cuestión sirve de base para seguir avanzando cautelosamente en la búsqueda de modelos contemporáneos de imputación y de sanciones adecuadas para las empresas que permitan frenar la generalización de una cultura corporativa que alienta a la violación de derechos humanos. Asimismo, el reconocimiento de la responsabilidad internacional penal de las empresas supone un esfuerzo para paliar los efectos socioeconómicos que se generan desproporcionalmente en el Sur Global a raíz del actual modelo económico global.

A nivel nacional, dado que muchos Estados han superado los obstáculos doctrinales de conformidad con los principios de sus ordenamientos internos, la práctica de recurrir a la vía penal para hacer efectiva la responsabilidad de las empresas por violaciones de derechos humanos o, en su defecto, a los directivos o ejecutivos de las mismas, se consolida cada vez más. Sin embargo, en esta práctica surgen obstáculos relacionados con cuestiones competenciales, procesales y sobre la responsabilidad penal de las empresas matrices por delitos cometidos por filiales o socios comerciales en terceros Estados.

Entre los objetivos del tratado internacional sobre empresas y derechos humanos se encuentran mejorar y reforzar los mecanismos de responsabilidad de las empresas. En este sentido, se presenta como una oportunidad para dar un mayor impulso de la responsabilidad penal de las empresas por violaciones de derechos humanos, tanto a nivel internacional como nacional. El futuro tratado internacional no solo se constituye como una oportunidad para subsanar los errores del pasado respecto a la responsabilidad penal internacional de las empresas, sino también para estructurar el Derecho penal de los Estados hacia una posición mínima respecto a esta cuestión. El tratado debería definir los posibles actos y omisiones atribuibles a las empresas por la comisión o contribución a violaciones graves de derechos humanos, así como las sanciones a las que estarían sujetas. Asimismo, con base en la experiencia, podría definir medidas para reducir los obstáculos que implica la práctica de enjuiciar penalmente a las empresas a nivel nacional por actos ilícitos cometidos en sus operaciones globales.

\section{Biblografía $^{72}$}

Amnistía Internacional. A Criminal Enterprise? Shell's Involvement in Human Rights Violations in Nigeria in the 1990s. Londres: Amnesty International, 2017.

BATESMITH, Alex. Corporate Criminal Responsibility for War Crimes and Other Violations of International Humanitarian Law: the Impact of the Business and Human Rights Movement. En: HARVEY, Caroline; SUMMERS, James; WHITE, Nigel D. (Ed.). Contemporary Challenges to the Laws of War: Essays in Honour of Professor Peter Rowe. Cambridge: CUP, 2014. p. 285-312.

BERNAZ, Nadia. An Analysis of the ICC Office of the Prosecutor's Policy Paper on Case Selection and Prioritization from the Perspective of Business and Human Rights. Journal of International Criminal Justice, v. 15, n. 3, p. 527-542, 2017.

BERNAZ, Nadia. Corporate Criminal Liability under International Law. The New TV S.A.L. and Akhbar Beirut S.A.L. Cases at the Special Tribunal for Lebanon. Journal of International Criminal Justice, v. 13, n. 2, p. 313330, 2015.

CHIOMENTI, Cristina. Corporations and the International Criminal Court. En: DE SCHUTTER, Olivier (Ed.). Transnational Corporations and Human Rights. Oxford-Portland: Hart Publishing, 2006. p. 287-312.

CLAPHAM, Andrew. The Question of Jurisdiction under International Criminal Lawover Legal Persons: Lessons from the Rome Conference on an International

72 En lo que respecta a las fuentes distintas de la literatura legal, por ejemplo, jurisprudencia, véase, notas a pie de página. 
Criminal Court. En: KAMMINGA, Menno T. y ZIAZARIFI, Saman (Ed.). Liability of Multinational Corporations under International Law. La Haya-Londres-Boston: Kluwer Law International, 2000. p. 139-195.

DE JONGE, Alice. Transnational corporations and international law. Accountability in the global business environment. Cheltenham-Northampton: Edward Elgar, 2001.

ENNEKING, Liesbeth et al. Zorgplichten van nederlandse ondernemingen inzake internationaal maatschappelijk verantwoord ondernemen. Een rechtsvergelijkend en empirisch onderzoek naar de stand van het Nederlandse recht in het licht van de UN Guiding Principles. Ámsterdam: WODC, 2015.

FAIRLIE, Megan A. The Hidden Costs of Strategic Communications for the International Criminal Court. Texas International Law Journal, v. 51, n. 3, p. 281-319, 2016.

FERENCZ, Benjamin B. An International Jurisdiction for Corporate Atrocities: Observations of a Former Nuremberg War Crimes Prosecutor. Harvard International Law Journal, v. 57, p. 15-19, 2016.

HERNÁNDEZ ZUBIZARRETA, Juan. El Tribunal Internacional para las empresas transnacionales y los derechos humanos. América Latina en movimiento, p. 1415, 2016.

ICJ. Corporate Complicity and Legal Accountability; Report of the International Commission of Jurists Expert Legal Panel on Corporate Complicity in International Crimes, Criminal Law and International Crimes. Ginebra: ICJ, 2008.

ICJ. Proposals for Elements of a Legally Binding Instrument on Transnational Corporations and Other Business Enterprises. Ginebra: ICJ, 2016.

IVORY, Radha; JOHN, Anna. Holding Companies Responsible? The Criminal Liability of Australian Corporations for Extraterritorial Human Rights Violations. University of New South Wales Law Journal, v. 40, n. 3, p. 1175-119, 2017.

JÄGERS, Nicola. Corporate Human Rights Obligations: In Search of Accountability. Amberes: Intersentia, 2002.

KALECK, Wolfgang; SAAGE-MAAß, Miriam. Corporate Accountability for Human Rights Violations Amounting to International Crimes. The Status Quo and its Challenges. Journal of international Criminal Justice, v. 8, n. 3, p. 699-724, 2010.

KYRIAKAKIS, Joanna. Corporations before International Criminal Courts: Implications for the International Criminal Justice Project. Leiden Journal of International Law, v. 30, n. 1, p. 221-240, 2017.

LAMBERT, Caitlin. Environmental Destruction in Ecuador: Crimes against Humanity under the Rome Statute? Leiden Journal of International Law, v. 30, n. 3, p. 707-729, 2017.

MONGELARD, Éric. Responsabilidad civil de las empresas por violaciones del derecho internacional humanitario. International Review of the Red Cross, n. 863, p. 1-30, 2006.

PÉREZ-LEÓN ACEVEDO, Juan Pablo. The Close Relationship between Serious Human Rights Violations and Crimes against Humanity: International Criminalization of Serious Abuses. Anuario Mexicano de Derecho Internacional, v. 17, p. 145-186, 2017.

RAMASASTRY, Anita. Corporate Complicity: From Nuremberg to Rangoon - An Examination of Forced Labor Cases and Their Impact on the Liability of Multinational Corporations. Berkeley Journal of International Law, v. 20, n. 1, p. 91-159 2002.

Ramasastry, Anita; CASSELL, Douglass. White Paper: Options for a Treaty on Business and Human Rights. Notre Dame Journal of International \& Comparative Law, v. 6, n. 1, p. 1-50, 2015.

RAMASASTRY, Anita; THOMPSON, Robert C. Commerce, Crime and Conflict Legal Remedies for Private Sector Liability for Grave Breaches of International Law. A Survey of Sixteen Countries New Security Programme Economic Agendas and Civil Wars. Executive Summary. Oslo: FAFO, 2006.

REQUEJO ISIDRO, Marta. Responsabilidad civil y derechos humanos en EEUU: ¿el fin del ATS? InDret. Revista para el análisis del Derecho, n. 3, p. 1-38, 2011.

Scheffer, David. Corporate Liability under the Rome Statute. Harvard International Law Journal, v. 57, p. 36-39, 2016.

SKINNER, Gwynne; MCCORQUODALE, Robert; DE SCHUTTER, Olivier. The Third Pillar. Access to Judicial Remedies for Human Rights Violations by Transnational Business. Bruselas-Londres: ICAR-COREECCJ, 2013. 
SMITH, Susan L. Changing Corporate Environmental Behaviour: Criminal Prosecutions as a Tool of Environmental Policy. En: ECKERSLEY, Robyn (Ed.). Markets, the State and the Environment towards Integration. Melbourne: McMillan Education Australia, 1995. p. 261-274.

STEPHENS, Beth. Corporate Accountability: International Human Rights Litigation against Corporations in US Courts. En: KAMMINGA, Menno T.; ZIA-ZARIFI, Saman (Ed.). Liability of Multinational Corporations under International Law. La Haya-Londres-Boston: Kluwer Law International, 2000. p. 209-230.

VAN DEN HERIK, Larissa; DAM-DE JONG, Daniëlla. Revitalizing the Antique War Crime of Pillage: The Potential and Pitfalls of Using International Criminal Law to Address Illegal Resource Exploitation during Armed Conflict. Criminal Law Forum, v. 22, n. 3, p. 237,
2011.

VERMEULEN, Gert; DE BONDT, Wendy; RYCKMAN, Charlotte. Liability of legal persons for offences in the EU. Amberes: Maklu, 2012.

WATTAD, Mohammed Said-Alden. Natural Persons, Legal Entities, and Corporate Criminal Liability under the Rome Statute. UCLA Journal of International Law and Foreign Affairs, v. 20, n. 2, p. 391-424, 2016.

ZERK, Jennifer A. Towards a fairer and more effective system of domestic law remedies. A report prepared for the Office of the UN High Commissioner for Human Rights. Disponible en: http://www.ohchr.org/Documents/ Issues/Business/DomesticLawRemedies/StudyDomesticeLawRemedies.pdf. 
Para publicar na Revista de Direito Internacional, acesse o endereço eletrônico www.rdi.uniceub.br ou www.brazilianjournal.org.

Observe as normas de publicação, para facilitar e agilizar o trabalho de edição. 